\section{Association of uric acid levels before start of conditioning with mortality after allogeneic hematopoietic stem cell transplantation - a prospective, non-interventional study of the EBMT Transplant Complication Working Party}

Olaf Penack, ${ }^{1}$ Christophe Peczynski, ${ }^{2}$ Steffie van der Werf, ${ }^{3}$ Jürgen Finke, ${ }^{4}$ Arnold Ganser, ${ }^{5}$ Helene Schoemans, ${ }^{6}$ Jiri Pavlu, ${ }^{7}$ Riitta Niittyvuopio, ${ }^{8}$ Wilfried Schroyens, ${ }^{9}$ Leylagül Kaynar, ${ }^{10}$ Igor W. Blau, ${ }^{1}$ Walter van der Velden, ${ }^{11}$ Jorge Sierra, ${ }^{12}$ Agostino Cortelezzi, ${ }^{13}$ Gerald Wulf, ${ }^{14}$ Pascal Turlure, ${ }^{15}$ Montserat Rovira, ${ }^{16}$ Zubeydenur Ozkurt, ${ }^{17}$ Maria J. Pascual-Cascon, ${ }^{18}$ Maria C. Moreira, ${ }^{19}$ Johannes Clausen, ${ }^{20}$ Hildegard Greinix, ${ }^{21}$

Rafael F. Duarte ${ }^{22 *}$ and Grzegorz W. Basak ${ }^{23 \#}$

${ }^{1}$ Charité Universitätsmedizin Berlin, Berlin, Germany; ${ }^{2}$ EBMT Statistical Unit, Paris, France; ${ }^{3}$ EBMT Data Office, Leiden, the Netherlands; ${ }^{4}$ University of Freiburg, Freiburg, Germany; ${ }^{5}$ Hannover Medical School, Hannover, Germany; ${ }^{6}$ Department of Hematology, University Hospital Leuven and KU Leuven, Leuven, Belgium; ' Imperial College, London, UK; ${ }^{8} \mathrm{HUCH}$ Comprehensive Cancer Center, Helsinki, Finland; ${ }^{9}$ Antwerp University Hospital, Antwerp, Belgium; ${ }^{10}$ Erciyes University Medical Faculty, Kayseri, Turkey; ${ }^{11}$ Radboud University - Nijmegen Medical Centre, Nijmegen, the Netherlands; ${ }^{12}$ Hospital Santa Creu I Sant Pau, Barcelona, Spain; ${ }^{13}$ Fondazione IRCCS - Ca'Granda, Milan, Italy; ${ }^{14}$ Universitätsklinikum Göttingen, Göttingen, Germany; ${ }^{15} \mathrm{CHRU}$ Limoges, Limoges,

France; ${ }^{16} \mathrm{Hospital}$ Clinic, Barcelona, Spain; ${ }^{17}$ Gazi University Faculty of Medicine, Ankara, Turkey; ${ }^{18} \mathrm{Hospital}$ Regional de Málaga, Malaga, Spain; ${ }^{19}$ Instituto National do Cancer, Rio de Janeiro, Brazil; ${ }^{20}$ Elisabethinen-Hospital, Linz, Austria; ${ }^{21} \mathrm{LKH}$ - University Hospital Graz, Graz, Austria; ${ }^{22}$ Hospital Universitario Puerta de Hierro, Madrid, Spain and

${ }^{23}$ Medical University of Warsaw, Warsaw, Poland

"RFD and GWB contributed equally as co-senior authors.

\section{ABSTRACT}

U ric acid is a danger signal contributing to inflammation. Its relevance to allogeneic stem cell transplantation (alloSCT) derives from preclinical models where the depletion of uric acid led to improved survival and reduced graft-versus-host disease (GvHD). In a clinical pilot trial, peritransplant uric acid depletion reduced acute GvHD incidence. This prospective international multicenter study aimed to investigate the association of uric acid serum levels before start of conditioning with alloSCT outcome. We included patients with acute leukemia, lymphoma or myelodysplastic syndrome receiving a first matched sibling alloSCT from peripheral blood, regardless of conditioning. We compared outcomes between patients with high and low uric acid levels with univariate- and multivariate analysis using a cause-specific Cox model. Twenty centers from 10 countries reported data on 366 alloSCT recipients. There were no significant differences in terms of baseline co-morbidity and disease stage between the high- and low uric acid group. Patients with uric acid levels above median measured before start of conditioning did not significantly differ from the remaining in terms of acute GvHD grades II-IV incidence (Hazard ratio [HR] 1.5, 95\% Confidence interval [CI]: 1.0-2.4, $P=0.08)$. However, they had significantly shorter overall survival (HR 2.8, 95\% CI: 1.7-4.7, $P<0.0001)$ and progression free survival (HR 1.6, 95\% CI: 1.1-2.4, $P=0.025)$. Non-relapse mortality was significantly increased in alloSCT recipients with high uric acid levels (HR 2.7, 95\% CI: 1.4-5.0, $P=0.003)$. Finally, the incidence of relapse after alloSCT was increased in patients with higher uric acid levels (HR 1.6, 95\% CI: 1.0-2.5, $P=0.04)$. We conclude that high uric acid levels before the start of conditioning correlate with increased mortality after alloSCT.

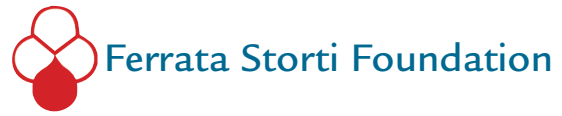

Haematologica 2020

Volume 105(7):1977-1983

\section{Correspondence:}

OLAF PENACK

olaf.penack@charite.de

Received: June 4, 2019.

Accepted: October 4, 2019.

Pre-published: October 10, 2019.

doi:10.3324/haematol.2019.228668

Check the online version for the most updated information on this article, online supplements, and information on authorship \& disclosures: www.haematologica.org/content/105/7/1977

\section{(C)2020 Ferrata Storti Foundation}

Material published in Haematologica is covered by copyright. All rights are reserved to the Ferrata Storti Foundation. Use of published material is allowed under the following terms and conditions:

https://creativecommons.org/licenses/by-nc/4.0/legalcode. Copies of published material are allowed for personal or internal use. Sharing published material for non-commercial purposes is subject to the following conditions:

https://creativecommons. org//icenses/by-nc/4.0/legalcode, sect. 3. Reproducing and sharing published material for commercial purposes is not allowed without permission in writing from the publisher. 


\section{Introduction}

High treatment-associated complication rates remain an important challenge to the success of allogeneic hematopoietic stem cell transplantation (alloSCT). A common mechanism of the major infectious- and non-infectious complications after alloSCT, such as graft-versus-host disease (GvHD) and sepsis, is severe inflammation. Critical to excess inflammation after alloSCT are host biomolecules termed 'endogenous danger signals', which can initiate and maintain non-infectious inflammatory responses by acting as pro-inflammatory mediators. ${ }^{1,2}$ One such molecule is uric acid. During conditioning for alloSCT, uric acid is released from injured cells and reduced renal clearance may contribute to high uric acid serum levels. ${ }^{3,4}$ Uric acid acts as a danger signal by enhancing T-cell responses via activation of the NOD-like receptor protein (NLRP) 3 inflammasome., ${ }^{5,6} \mathrm{~A}$ pre-clinical study has provided basic evidence on the regulation of GvHD by uric acid via Nlrp3 inflammasome-mediated IL-1 production. The significance of uric acid for alloSCT outcome has been underlined by results from a pilot study reporting reduced incidence of acute GvHD in patients undergoing alloSCT after depletion of uric acid using urate oxidase. ${ }^{8}$

Based on these results, and because uric acid is a routine laboratory parameter assessed in patients undergoing alloSCT, there is a strong rationale to investigate the use of uric acid as a biomarker and possibly as a therapeutic target during alloSCT. To study the role of uric acid levels in alloSCT, the Transplant Complications Working Party (TCWP) of the European Society for Blood and Marrow Transplantation (EBMT) performed a prospective, multicenter, non-interventional study. We hypothesized that a high uric acid level prior to alloSCT is an independent risk factor for increased mortality and for the development of severe acute GvHD.

\section{Methods}

\section{Data source, study design and data collection}

We asked EBMT centers performing more than 50 alloSCT per year if they were willing to participate in this prospective study. Twenty centers in ten countries agreed to participate. Data collection for the EBMT registry was approved by the institutional review board and/or Ethics Committee in all centers. Data were prospectively collected between August, 2014 and February, 2018. Consecutive alloSCT recipients with acute leukemia, lymphoma or myelodysplastic syndrome (MDS) receiving a first matched sibling alloSCT from peripheral blood, regardless of conditioning, were eligible, provided they had signed an informed consent document that permitted sharing of clinical data according to national rules. Basic data on patient and disease characteristics as well as longer term follow-up was taken from minimal essential data (MED-A) forms, which are submitted from all consecutive patients to the central EBMT registry. In addition, we designed registration and MED-B/C forms that were prospectively collected and specific to this study (see the Online Supplementary Materials and Methods). The MED-B/C form contained detailed information on uric acid serum levels prior to alloSCT, patient characteristics, infectious- as well as non-infectious complications, GvHD staging, morbidity and mortality. Uric acid levels were determined at the time of hospital admission for alloSCT directly before the start of conditioning therapy. Treatment teams completed the specific forms at the time of registration and at day +100 after alloSCT.

\section{Endpoints and statistical analyses}

Patient, disease, and transplant-related characteristics for the two cohorts (uric acid levels prior to alloSCT above median / uric acid levels below median) were compared by using 2 statistics for categorical variables and the Mann-Whitney test for continuous variables. The primary endpoint was the incidence of acute GvHD. Secondary endpoints were relapse incidence (RI), nonrelapse mortality (NRM), overall survival (OS), progression free survival (PFS) and the incidence of chronic GvHD. PFS was defined as survival with no evidence of relapse or progression. RI was defined as the probability of having had a relapse during follow-up time. Death without experiencing a relapse was a competing event. NRM was defined as death without evidence of relapse or progression. OS was defined as the time from alloSCT to death, regardless of the cause. Acute GvHD was graded according to the modified Seattle-Glucksberg criteria ${ }^{9}$ and chronic GvHD according to the revised Seattle criteria. ${ }^{10}$ Cumulative incidence was used to estimate the endpoints of NRM, RI, acute and chronic GvHD to accommodate for competing risks. To study acute and chronic GvHD, we considered relapse and death to be competing events. Probabilities of OS and PFS were calculated using the KaplanMeier method. Univariate analyses were done using the Gray test for cumulative incidence functions and the log rank test for OS and PFS. A Cox proportional hazards model was used for multivariate regression. All variables differing significantly between the two groups or factors associated with one outcome in univariate analysis were included in the Cox model. The following variables entered the multivariate models as possible confounders: age, sex mismatch between recipient and donor, diagnosis, disease status, Karnofsky score, number of CD34 cells given, intensity of conditioning (EBMT definition: myeloablative conditioning (MAC) was defined as total body irradiation (TBI) $>6$ Grey or oral busulfan $>8$ $\mathrm{mg} / \mathrm{kg}$ or intravenous busulfan $>6.4 \mathrm{mg} / \mathrm{kg}$ ), type of GvHD prophylaxis, ATG use, time from diagnosis to transplant, year of transplant and CMV status. As the number of variables was too high regarding the number of events, a stepwise selection using Akaike information criterion (AIC) was run for all the confounding factors. The difference between the two cohorts was then assessed in the final selected model.

Results were expressed as the hazard ratio (HR) with the 95\% confidence interval (95\% CI). Proportional hazards assumptions were checked systematically for all proposed models using the Grambsch-Therneau residual-based test. All tests were two-sided. The type I error rate was fixed at 0.05 for the determination of factors associated with time-to-event outcomes. Statistical analyses were performed in November 2018 with R 3.4.2 (R Core Team (2017). R: A language and environment for statistical computing. $R$ Foundation for Statistical Computing, Vienna, Austria. URL https://www.R-project.org/.)

\section{Results}

\section{Patients, transplant characteristics and uric acid measurement}

The entry criteria for analysis of primary and secondary endpoints were fulfilled in 386 patients. We used the last uric acid serum level that was masured in the individual patients before start of conditioning. The median time point of measurement was three days before start of conditioning. The range was between 0 days (morning before start of conditioning) to 22 days before conditioning. The main patients and transplant characteristics that were included in the analysis of OS are described in Table 1. Most parameters were balanced between the two cohorts. 
Table 1. Patient characteristics.

\begin{tabular}{|c|c|c|c|}
\hline & Uric acid $\leq 4.3 \mathrm{mg} / \mathrm{dL}$ ( $\mathrm{n=186}$ ) & Uric acid >4.3 mg/dL (n=180) & $P$ \\
\hline Year of transplant median (range) [IQR] & 2015 (2014-2018) [2015-2017] & 2015 (2014-2018) [2014-2016] & 0.10 \\
\hline Patient age (years) median(range) & $52(17-71)$ & $55(20-71)$ & 0.11 \\
\hline $\begin{array}{l}\text { Time from diagnosis to transplant } \\
\text { (months) median (range) }\end{array}$ & $4(1-61)$ & $5(1-71)$ & 0.09 \\
\hline \multicolumn{4}{|l|}{ Sex mismatch } \\
\hline Female to male & $30(17 \%)$ & $52(29 \%)$ & 0.005 \\
\hline Other combination & $148(83 \%)$ & $125(71 \%)$ & \\
\hline Missing & 8 & 3 & \\
\hline \multicolumn{4}{|l|}{ Diagnosis } \\
\hline Acute leukemia & $130(70 \%)$ & $118(66 \%)$ & 0.7 \\
\hline Lymphoma & $17(9 \%)$ & $19(10 \%)$ & \\
\hline MDS & $39(21 \%)$ & $43(24 \%)$ & \\
\hline \multicolumn{4}{|l|}{ Disease status } \\
\hline $\mathrm{CR}$ & $120(66 \%)$ & $125(72 \%)$ & 0.2 \\
\hline Not in CR & $61(34 \%)$ & $48(28 \%)$ & \\
\hline Missing & 5 & 7 & \\
\hline DRI & & & 0.7 \\
\hline Low & $8(4 \%)$ & $7(4 \%)$ & \\
\hline Intermediate & $107(60 \%)$ & $105(63 \%)$ & \\
\hline High & $51(30 \%)$ & $49(30 \%)$ & \\
\hline Very high & $10(6 \%)$ & $5(3 \%)$ & \\
\hline Missing & 10 & 14 & \\
\hline \multicolumn{4}{|l|}{ ATG } \\
\hline No & $100(54 \%)$ & $106(59 \%)$ & 0.3 \\
\hline Yes & $86(46 \%)$ & $74(41 \%)$ & \\
\hline \multicolumn{4}{|l|}{ Conditioning intensity } \\
\hline MAC/Chemo & $46(25 \%)$ & $37(21 \%)$ & 0.2 \\
\hline MAC/TBI & $36(20 \%)$ & $26(15 \%)$ & \\
\hline RIC & $102(55 \%)$ & $115(64 \%)$ & \\
\hline Missing & 2 & 2 & \\
\hline \multicolumn{4}{|l|}{ GvHD prophylaxis } \\
\hline Calcineurin inhibitor + MMF & $85(48 \%)$ & $84(47 \%)$ & 0.6 \\
\hline Calcineurin inhibitor + MTX & $64(36 \%)$ & $71(40 \%)$ & \\
\hline Calcineurin inhibitor mono & $27(15 \%)$ & $22(12 \%)$ & \\
\hline Missing & 10 & 3 & \\
\hline Donor/patient CMV & & & 0.2 \\
\hline$-/$ & $28(16 \%)$ & 38 (21\%) & \\
\hline Other & $146(83 \%)$ & $136(78 \%)$ & \\
\hline Missing & 12 & 6 & \\
\hline Karnofsky score & & & 0.14 \\
\hline $10-80$ & $38(21 \%)$ & $27(15 \%)$ & \\
\hline $90-100$ & $140(78 \%)$ & $149(84 \%)$ & \\
\hline Missing & 8 & 4 & \\
\hline $\mathrm{HT}-\mathrm{CI}=0$ & $64(50 \%)$ & $56(40 \%)$ & 0.19 \\
\hline HT-CI = 1 or 2 & $20(15 \%)$ & $32(23 \%)$ & \\
\hline $\mathrm{HT}-\mathrm{CI} \geq 3$ & $45(35 \%)$ & $51(37 \%)$ & \\
\hline Missing & 57 & 41 & \\
\hline
\end{tabular}

MDS: myelodysplastic syndrome; IQR: interquartile range; CR: complete remission; DRI: disease risk index; ATG: anti-thymocyte globulin; MAC: myeloablative conditioning; RIC: reduced intensity conditioning; TBI: total body irradiation; GvHD: graft-versus-host disease; MMF: Mycophenolate mofetil; MTX: Methotrexate; CMV: cytomegalovirus; HT-CI: hematopoietic cell transplantation cumulative incidence. 
A

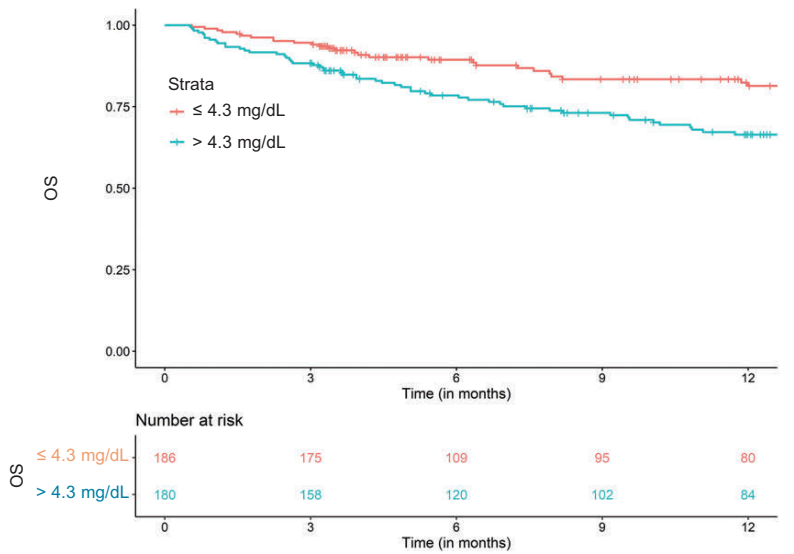

Figure 1. Survival at one year after allogeneic stem cell transplantation. Overall survival (OS) (A) and progression free survival (PFS) (B) after allogeneic stem cell transplantation (alloSCT) in cohorts according to uric acid serum levels prior to alloSCT: with high uric acid serum levels (blue line), patients with low uric acid levels (red line).

However, a higher percentage of sex mismatch transplants in the direction of female donor to male recipient was observed in the group of patients with uric acid above median before alloSCT. The frequency of patients with reduced-intensity conditioning (RIC) versus myeloablative conditioning (MAC) did not differ significantly between the high uric acid group and the low uric acid group $(P=0.077)$. When looking exclusively at TBI $(P=0.59)$ or by mixing conditioning intensity and TBI (MAC/Chemo vs. MAC/TBI vs. RIC: $P=0.20)$, no statistically significant difference could be observed.

\section{Incidence of acute and chronic GvHD}

In the present study, the incidence of acute GvHD grades II-IV and grades III-IV in the whole population at 100 days were $25 \%$ and $11 \%$, respectively. We observed no significant differences in the frequency of clinically significant acute GvHD grades II-IV in univariate (HR 1.2, 95\% CI: 0.8-1.9, $P=0.4$ ) and multivariate (HR 1.5, 95\% CI: $1.0-2.4, P=0.08)$ analysis comparing the high-versus the low uric acid cohorts. (Table 2 and Table 3). We found no significant differences in the frequency of acute GvHD grades III-IV in univariate $(\mathrm{HR}=1.8,95 \% \mathrm{CI}: 0.9-3.4$, $P=0.08$ ) and multivariate (HR 1.7, 95\% CI: 0.9-3.2, $P=0.11$ ) analysis in alloSCT recipients with uric acid levels above cut-off before transplantation versus those with low uric acid levels (Table 2 and Table 3).

In the whole population, the incidence of chronic $\mathrm{GvHD}$ at one year and two years was $27 \%$ and $40 \%$, respectively. The incidence of severe chronic GvHD at 1 year and 2 years was $18 \%$ and $24 \%$, respectively. We observed no differences in incidence and severity of chronic GvHD between the two cohorts (Table 3 and Table 4). The chronic GvHD incidence was significantly lower in alloSCT recipients receiving anti-T-cell globulin (ATG) as part of the conditioning regimen (ATG, HR 0.25 95\% CI: 0.13-0.5, $P<0.0001)$.

\section{Survival endpoints}

The median follow-up time was 15.2 months (95\% CI: 13.1-17.0) and $O S$ in the whole population at 1 year was
Table 2. Univariate global comparison of acute graft-versus-host disease shown at day +100 after allogeneic stem cell transplantation.

\begin{tabular}{lcc} 
Group & $\begin{array}{l}\text { Acute Gv/D } \\
\text { II-IV (95\% CI) }\end{array}$ & $\begin{array}{l}\text { Acute GvHD } \\
\text { III-IV (95\% CI) }\end{array}$ \\
Uric acid $</=4.3 \mathrm{mg} / \mathrm{dL}$ & $22 \%(17-29)$ & $8 \%(5-13)$ \\
Uric acid $>4.3 \mathrm{mg} / \mathrm{dL}$ & $26 \%(20-33)$ & $14 \%(9-19)$ \\
\hline$P$-value & 0.39 & 0.08 \\
\hline
\end{tabular}

GvHD: graft-versus-host disease; Cl: confidence interval.

$74 \%$. We found, that OS and PFS of alloSCT recipients with uric acid levels above cut-off, measured before start of conditioning, were significantly shorter compared with the low uric acid cohort (Figure 1A, OS univariate HR 2.4, 95\% CI: 1.6-3.7, $P<0.001$; multivariate HR 2.8, 95\% CI: 1.7-4.7, $P<0.0001$ ) (Figure 1B, PFS univariate HR 2.0, 95\% CI: 1.1-3.7, $P=0.02$; multivariate HR 2.7, 95\% CI: 1.4-5.0, $P=0.003)$. Non-relapse mortality was significantly increased in alloSCT recipients with high uric acid levels prior to start of conditioning (Figure 2A, univariate HR 2.0, 95\% CI: 1.1-3.7, $P=0.02$; multivariate HR 2.7, 95\% CI: 1.4 5.0, $P=0.003)$. Multivariate as well as univariate analyses are shown in Table 3 and Table 4 . We conclude that serum uric acid levels prior to alloSCT are an independent risk factor for mortality.

At the last follow-up $29.3 \%$ of the patients were dead (49.6\% from relapse, $45.2 \%$ alloSCT-related causes and $2.6 \%$ from other causes). The causes of death in patients without relapse were mainly due to infection. A descriptive analysis of the causes of death is given in Table 5 .

\section{Incidence of infections}

We first looked at the incidence of infections in both cohorts and found no significant differences (multivariate HR 0.8, 95\% CI: 0.6-1.1, P=0.4). We than looked at infection-related mortality and found a significant higher number of infection-related deaths in the high uric acid group (Figure 2C, multivariate HR 2.6, 95\% CI: 1.2-5.2, $P=0.01$ ).

\section{Incidence of relapse}

Since uric acid is known to be released from malignant 
A

NRM - Uric acid

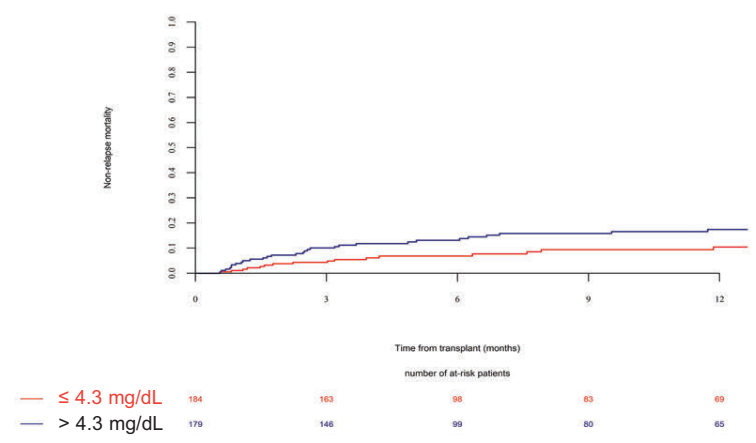

C

Infection-related mortality - Uric Acid

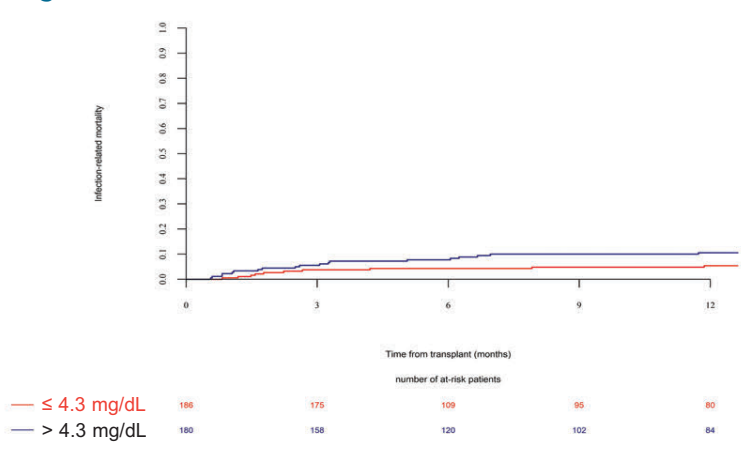

B RI - Uric acid

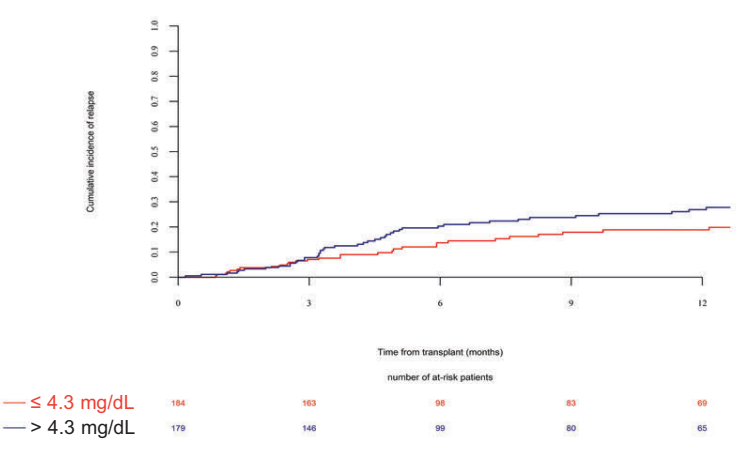

Figure 2. Non-relapse mortality, relapse incidence and infection-related mortality at one year after allogeneic stem cell transplantation. Incidence of non-relapse mortality (NRM) (A), relapse incidence (RI) (B) and infection-related mortality $(\mathrm{C})$ after allogeneic stem cell transplantation (alloSCT) in cohorts according to uric acid serum levels prior to alloSCT: patients with high uric acid serum levels (blue line), patients with low uric acid levels (red line).

Table 3. Multivariate global comparison.

\begin{tabular}{lccc} 
Uric acid $>4.3 \mathrm{mg} / \mathrm{dL}$ & HR & $95 \% \mathrm{Cl}$ & P \\
versus $\leq 4,3 \mathrm{mg} / \mathrm{dL}$ & & $1,72-4,68$ & $<0.0001$ \\
\hline Overall Survival & 2,83 & $1,06-2,39$ & 0.03 \\
Progression Free Survival & 1,59 & $1,02-2,49$ & 0.04 \\
\hline Relapse Incidence & 1,59 & $1,41-5,01$ & 0.003 \\
Non-Relapse Mortality & 2,65 & $0,63-1,45$ & 0.84 \\
\hline Chronic GvHD & 0,96 & $0,62-1,88$ & 0.79 \\
Extensive Chronic GvHD & 1,08 & $0,95-2,43$ & 0.08 \\
\hline Acute GvHD II-IV & 1,52 & $0,88-3,19$ & 0.11 \\
Acute GvHD III-IV & 1,68 & & \\
\hline
\end{tabular}

GvHD: graft-versus-host disease; HR: hazard ratio; Cl: confidence interval.

tumours, we were interested in a possible connection of uric acid levels and disease relapse. We found that the relapse incidence in all patients at 1 year was 13\%. The relapse incidence was moderately increased in the cohort with higher uric acid levels (Figure 2B, Table 3 and Table 4, univariate HR 1.6, 95\% CI: 1.0-2.5; $P=0.09$; multivariate HR 1.6, 95\% CI: 1.0-2.5, P=0.04).

\section{Discussion}

This prospective study identifies uric acid levels, taken before start of conditioning therapy, as a laboratory biomarker to predict mortality after alloSCT.
In animal experiments and in a clinical pilot study the depletion of uric acid with rasburicase led to a reduced frequency of severe GvHD.,8 Therefore, our initial hypothesis was to primarily find an association between uric acid with acute GvHD and then secondarily with NRM. However, we found a strong positive association of uric acid levels with mortality after alloSCT whereas the association between uric acid levels and acute GvHD incidence was not statistically significant. One of the reasons could be the low incidence of GvHD in our cohort due to the patient characteristics of human leukocyte antigen (HLA)-identical sibling transplant with a high proportion of anti-thymocyte globulin (ATG) use. The use of in vivo T-cell depletion with ATG in HLA-identical sibling 
Table 4. Univariate global comparison of overall survival, non-relapse mortality, relapse incidence as well as chronic graft-versus-host disease incidence and severity at one year after allogeneic stem cell transplantation.

\begin{tabular}{|c|c|c|c|c|c|c|}
\hline Group & $\begin{array}{c}\text { OS } \\
(95 \% \text { CI) }\end{array}$ & $\begin{array}{c}\text { PFS } \\
(95 \% \text { CI) }\end{array}$ & $\begin{array}{c}\text { RI } \\
(95 \% \text { Cl) }\end{array}$ & $\begin{array}{l}\text { NRM } \\
(95 \% \text { CI) }\end{array}$ & $\begin{array}{l}\text { CGVHD } \\
\text { (95\% CI) }\end{array}$ & $\begin{array}{c}\text { Extensive cGvHD } \\
\text { (95\% Cl) }\end{array}$ \\
\hline Uric acid $</=4.3 \mathrm{mg} / \mathrm{dL}$ & $\begin{array}{c}81 \% \\
(75-88)\end{array}$ & $\begin{array}{c}71 \% \\
(64-79)\end{array}$ & $\begin{array}{c}19 \% \\
(13-26)\end{array}$ & $\begin{array}{c}10 \% \\
(6-16)\end{array}$ & $\begin{array}{c}28 \% \\
(20-36)\end{array}$ & $\begin{array}{c}16 \% \\
(10-24)\end{array}$ \\
\hline $\begin{array}{l}\text { Uric acid } \\
>4.3 \mathrm{mg} / \mathrm{dL}\end{array}$ & $\begin{array}{c}66 \% \\
(59-74)\end{array}$ & $\begin{array}{c}56 \% \\
(48-64)\end{array}$ & $\begin{array}{c}27 \% \\
(20-34)\end{array}$ & $\begin{array}{c}17 \% \\
(12-24)\end{array}$ & $\begin{array}{c}26 \% \\
(19-33)\end{array}$ & $\begin{array}{c}18 \% \\
(12-25)\end{array}$ \\
\hline$P$-value & $<0.001$ & 0.001 & 0.09 & 0.02 & 0.43 & 0.85 \\
\hline
\end{tabular}

OS: overall survival; PFS: progression free survival; NRM: non-relapse mortality; RI: relapse incidence: cGvHD: chronic graft-versus-host disease; CI: confidence intervall.

alloSCT in European centres increased recently. ${ }^{11}$ This trend is reflected by a higher frequency of ATG use in the present study $(41-46 \%)$ than we had initially expected. Another likely reason is a hyperinflammatory status connected to high uric acid levels not restricted to acute GvHD with a negative impact on different relevant clinical situations after alloSCT, such as sepsis ${ }^{12}$ and adult respiratory distress syndrome (ARDS). ${ }^{13}$ This view is supported by our observation that death due to both infectious and non-infectious complications after alloSCT was increased in patients with high uric acid levels. Interestingly, an association of uric acid levels with decreased solid organ transplant survival has been described further underlining the significance of uric acid for transplantation biology. ${ }^{14,15}$ Our study extends these findings to the setting of alloSCT.

Our study contradicts results from a previous retrospective single centre study, which did not show any significant association of uric acid levels prior to transplantation to mortality after alloSCT. ${ }^{16}$ Differences in study designs between the current larger, multicentre and prospective study versus the already published smaller, single centre and retrospective study may be the reason for the differing results.

A limitation of this clinical study is the lack of mechanistic insight on the role of uric acid in development of complications after alloSCT. The study design did not allow the investigation of whether the impaired outcome in the high urid acid cohort is due to an active role of uric acid in causing inflammation or whether it is because uric acid primarily is a surrogate biomarker reflecting a hyperinflammatory status and/or activity of the underlying malignancy. However, we found no significant differences regarding the disease status as well as disease risk index (DRI) (complete remission [CR] vs. non-CR) in between the two groups (high uric acid vs. low uric acid). In addition there were no significant differences in the performance status as well as the alloSCT-CI in between the two groups. This data is no proof, but rather points in the direction that high uric acid values were not merely a reflection of advanced disease or increased co-morbidity in our study.

A further limitation of our results is that our patient population was restricted to alloSCT from HLA-identical sibling donors. We are therefore unable to draw definite
Table 5. Causes of death in both cohorts.

\begin{tabular}{lcc} 
& $\begin{array}{c}\text { Uric acid } \leq 4.3 \\
(\mathbf{n = 1 8 6}) \mathbf{m g} / \mathbf{d L}\end{array}$ & $\begin{array}{c}\text { Uric acid >4.3 } \\
\mathbf{m g} / \mathrm{dL} \text { ( }(\mathbf{n = 1 8 0})\end{array}$ \\
Alive at last follow up & $155(83.3 \%)$ & $106(58.9 \%)$ \\
Dead & $31(16.7 \%)$ & $74(41.1 \%)$ \\
\hline Dead due to relapse & $13(6.9 \%)$ & $38(21.1 \%)$ \\
Dead without relapse & $18(9.7 \%)$ & $36(20.0 \%)$ \\
\hline Dead due to infection & $5(3.2 \%)$ & $20(11.1 \%)$ \\
Dead due to GrHD & $2(1.0 \%)$ & $10(5.6 \%)$ \\
\hline Dead due to infection and GrHD & $3(1.6 \%)$ & $5(2.8 \%)$ \\
Dead due to other causes or unknown & $8(4.3 \%)$ & $1(0.5 \%)$
\end{tabular}

GvHD: graft-versus-host disease.

conclusions from these results regarding the association of uric acid levels with outcome in matched unrelated donor alloSCT or in haploidentical alloSCT, which are increasingly used. Another limitation is the incomplete understanding of the effects of uric acid on post alloSCT immunity. While previous studies showed that uric acid leads to a inflammatory status in leukocytes and our results demonstrate impaired survival in alloSCT recipients with uric acid levels above median, it may be premature to conclude that uric acid is a negative factor at any time around alloSCT. There are clinical situations where immune activation is necessary and desired - e.g. in anti-infectious and anti-tumor immunity. The impact of depleting uric acid on immune activity in these situations remains to be determined. In addition, it is possible that uric acid is involved in immune reconstitution as it has been suggested recently that changes in uric acid serum levels can indicate incipient or remaining immunological activity after SCT or induction therapy in patients with hematologic malignancies. $^{17}$

In conclusion, this study supports the use of serum uric acid levels as biomarker for alloSCT outcome.

\section{Acknowledgments}

$J P$ acknowledges the support of the Imperial NIHR-BCR. We acknowledge support from the German Research Foundation (DFG) and the Open Access Publication Funds of Charite Universitätsmedizin Berlin. 


\section{References}

1. Apostolova $\mathrm{P}$, Zeiser $\mathrm{R}$. The role of purine metabolites as DAMPs in acute graft-versus-host disease. Front Immunol. 2016; 7:439.

2. Zeiser R, Blazar BR. Acute graft-versus-host disease - biologic process, prevention, and therapy. N Engl J Med. 2017;377(22):21672179.

3. Cannell PK, Herrmann RP. Urate metabolism during bone marrow transplantation. Bone Marrow Transplant. 1992;10(4):337-339.

4. Joo SH, Park JK, Lee EE, et al. Changes in serum uric acid levels after allogeneic hematologic stem cell transplantation: A retrospective cohort study. Blood Res. 2016;51(3):200-203.

5. Martinon F, Petrilli V, Mayor A, et al. Goutassociated uric acid crystals activate the NALP3 inflammasome. Nature. 2006; 440(7081):237-241.

6. Braga TT, Forni MF, Correa-Costa M, et al. Soluble uric acid activates the NLRP3 inflammasome. Sci Rep. 2017;7:39884.
7. Jankovic D, Ganesan J, Bscheider M, et al. The Nlrp3 inflammasome regulates acute graft-versus-host disease. J Exp Med 2013;210(10):1899-1910

8. Yeh AC, Brunner AM, Spitzer TR, et al. Phase I study of urate oxidase in the reduction of acute graft-versus-host disease after myeloablative allogeneic stem cell transplantation. Biol Blood Marrow Transplant. 2014;20(5):730-734.

9. Przepiorka D, Weisdorf D, Martin P, et al 1994 Consensus conference on acute GVHD grading. Bone Marrow Transplant. 1995;15(6):825-828.

10. Lee SJ, Vogelsang G, Flowers ME. Chronic graft-versus-host disease. Biol Blood Marrow Transplant. 2003;9(4):215-233.

11. Kroger N, Solano C, Wolschke C, Bandini $\mathrm{G}$, et al. Antilymphocyte globulin for prevention of chronic graft-versus-host disease. N Engl J Med. 2016;374(1):43-53.

12. Pehlivanlar-Kucuk M, Kucuk AO, et al. The association between serum uric acid leve and prognosis in critically Ill patients, uric acid as a prognosis predictor. Clin Lab.
2018;64(9):1491-1500.

13. Lee HW, Choi SM, Lee J, et al. Serum uric acid level as a prognostic marker in patients with acute respiratory distress syndrome. Intensive Care Med. 2019;34(5):404-410

14. Asleh R, Prasad M, Briasoulis A, et al. Uric acid is an independent predictor of cardiac allograft vasculopathy after heart transplantation. J Heart Lung Transplant. 2018, 37(9):1083-1092.

15. Kim DG, Choi HY, Kim HY, et al. Association between post-transplant serum uric acid levels and kidney transplantation outcomes. PloS One. 2018; 13(12):e0209156.

16. Ostendorf BN, Blau O, Uharek L, et al Association between low uric acid levels and acute graft-versus-host disease. Ann Hematol. 2015;94(1):139-144.

17. Haen SP, Eyb V, Mirza N, et al. Uric acid as a novel biomarker for bone-marrow function and incipient hematopoietic reconstitution after aplasia in patients with hematologic malignancies. J Cancer Res Clin Oncol. 2017;143(5):759-771. 